\title{
Análisis energético de un aerogenerador con diferentes grados de inclinación del aspa usando integral numérica
}

\author{
Energy analysis of a wind turbine with different blade inclination angle using \\ numerical integral
}

JUÁREZ-TOLEDO, Carlos†', MARTÍNEZ-CARRILLO, Irma* y HERNÁNDEZ-EPIGMENIO, Miguel Ángel

Universidad Autónoma del Estado de México

ID $1^{\text {er }}$ Autor: Carlos, Juárez-Toledo / ORC ID: 0000-0002-7440-3246, Researcher ID Thomson: C-1368-2016, CVU CONACYT ID: 39912

ID $1^{\text {er }}$ Coautor: Irma, Martínez-Carrillo / ORC ID: 0000-0002-7952-4418, Researcher ID Thomson: B-9264-2016, CVU CONACYT ID: 39914

ID $2^{\text {do }}$ Coautor: Miguel Ángel, Hernández-Epigmenio / ORC ID: 0000-0002-1683-4080, Researcher ID Thomson: F-95142018, CVU CONACYT ID: 786771

DOI: $10.35429 / J T E N .2020 .13 .4 .9 .15$

Recibido 03 de Marzo, 2020; Aceptado 30 Junio, 2020

\section{Resumen}

Las innovaciones tecnológicas en las energías renovables han ayudado para su mejor implementación, una de las principales áreas de crecimiento en el tema es el desarrollo sistemas que hagan posible su implementación sin depender de las condiciones meteorológicas del lugar de implementación. Un claro ejemplo son los aerogeneradores, los cuales casi siempre son diseñados para obtener su máximo par de giro, lo cual lleva consigo una gran ventaja de producción de energía eléctrica a bajas velocidades de viento, pero contiene el inconveniente que si se ubica en lugares con velocidades de viento promedio superiores para los que fue diseñado podría llegar a dañar al equipo. En el artículo se realiza una evaluación de la eficiencia de un aerogenerador horizontal de tres álabes de 10, 20 y 40 grados de inclinación. La contribución lograda en el artículo es mostrar que por medio de integración numérica es posible determinar el rendimiento de un aerogenerador, para tal propósito se utilizan diferentes inclinaciones del alabe.

Aerogenerador, Diseño de Aspas, Eficiencia de un Sistema Eólico, Inclinación de Aspas, Integración Numérica

\begin{abstract}
The increase of renewable energy technologies provides a better way of implementation. One of the main problems for the renewable energy is that it depends of the local place topology or the weather conditions. Wind turbines are designed to obtain their maximum torque, this has the advantage of generating electricity with low air speed, but it has the disadvantage that it cannot be used in places with higher wind speeds, because the device can be broken. In the paper, an evaluation was made of the efficiency of a horizontal wind turbine of three blades with 10, 20 and 40 degrees of inclination, energy expenditure was obtained by means numerical integration. The purpose this article is to determine that the change of inclination of the blade has a direct influence on the performance of the wind turbine.
\end{abstract}

Wind Turbine, Blade Design, Efficiency of a Wind System, Inclination of Blades, Numerical Integration

Citación: JUÁREZ-TOLEDO, Carlos, MARTÍNEZ-CARRILLO, Irma y HERNÁNDEZ-EPIGMENIO, Miguel Ángel. Análisis energético de un aerogenerador con diferentes grados de inclinación del aspa usando integral numérica. Revista de Ingeniería Tecnológica. 2020. 4-13: 9-15

\footnotetext{
* Correspondencia del Autor (Correo electrónico: imartinezca@uaemex.mx)

$\dagger$ Investigador contribuyendo como primer autor.
} 


\section{Introducción}

La creciente demanda de energía y la conciencia ambiental han provocado interés en la energía eólica. Como resultado, el viento es la fuente de energía de más rápido crecimiento en el mundo hoy. Los marcos de políticas y los planes de acción ya han sido formulados en varios rincones para satisfacer al menos el 20 por ciento de la demanda mundial de energía con las nuevas energías renovables para 2010, entre los cuales el viento será el principal jugador [Mathew, 2006).

La generación eólica juega un rol muy importante para la red eléctrica, pero tiene el inconveniente de que no siempre se puede garantizar el flujo constante o suficiente de suministro de viento, debido a que se deben consideran diversas variables ambientales para poder predecir el clima (Akinci, 2013). Bakker muestra que estimar las tendencias de las velocidades del viento para aumentar el rendimiento de los aerogeneradores es un proceso complejo (Bakker, 2012).

En México existe un corredor muy importante en la Venta, Oaxaca, donde se encuentran instaladas las principales centrales eólicas (Sener, 2016). Hasta el año 2017 en México existían más de 4000 MW producidos por energía eólica en un total de 42 parques (Asociación Mexicana de Energía Eólica, 2017). Los resultados promedio del estudio de las velocidades del viento de la Venta son (Cadenas, 2008):

Moda tipo A: vientos moderados $(0 \mathrm{~m} / \mathrm{s}$ a 10 $\mathrm{m} / \mathrm{s})$.

Moda tipo B: vientos fuertes $(10 \mathrm{~m} / \mathrm{s}$ a $25 \mathrm{~m} / \mathrm{s})$.

Contrastando en velocidad con otros estudios, por ejemplo, el realizado en el estado de Veracruz donde promedio de velocidad es de $5.45 \mathrm{~m} / \mathrm{s}$ (Hernández, 2011), lo cual es una velocidad significativamente menor a la zona de La Venta. La energía en los parques eólicos ha venido creciendo, pero también la de baja potencia, debido a que es una buena alternativa económica de electricidad para sitios aislados.

Como puede notarse en México existe potencial para la generación de la energía eléctrica a través de las grandes plantas de generación eólicas, así como de las de baja potencia.
Pero las diferentes variaciones de velocidades en las rachas de viento hacen que sea necesario un método confiable para seleccionar el tipo de aerogenerador que consiga la mejor eficiencia según las características de la zona.

En la actualidad se busca hacer que los aerogeneradores sean lo más eficientes posibles (Tavner, 2007), buscando que generen electricidad desde el mínimo posible de velocidad del viento, hasta que soporten las máximas ráfagas posibles. Convencionalmente, los aerogeneradores de alta potencia están diseñados para soportar rachas de viento de 28 hasta $30 \mathrm{~m} / \mathrm{s}$, mientras un valor típico para que actúe el freno mecánico de los aerogeneradores de baja potencia es de aproximadamente en los 20 a $22 \mathrm{~m} / \mathrm{s}$.

Una manera de variar la eficiencia de los aerogeneradores para soportar una mayor velocidad sin que el freno mecánico se vea comprometido es por medio del ángulo de inclinación de entrada de flujo de aire.

En este artículo se presenta un estudio de una turbina de baja velocidad de un álabe de 10 , 20 y 40 grados de inclinación, mostrando su eficiencia ante diferentes velocidades. Para tal fin se diseñó un prototipo de túnel de viento con la finalidad de obtener un flujo laminar y someter al álabe a diferentes velocidades de viento.

Las variables que se analizan para determinar la eficiencia de cada tipo de aspa son la velocidad angular de la turbina, la cual se mide directamente con un enconder o codificador rotatorio compuesto por un optoacoplador, y la velocidad del viento por medio de un sensor conectado dentro del túnel de viento.

\section{Potencia y torque de la turbina eólica}

La energía por unidad de tiempo de una turbia puede expresarse por la siguiente ecuación (Richmond-Navarro, 2016).

$P=\frac{1}{2} \rho_{a} A_{T} V^{3}$

donde $\rho_{a}$ es la densidad del aire, $A_{T}$ es el área de la sección transversal del rotor y $V$ es la velocidad del viento ver figura 1, (Mathew, 2006). 


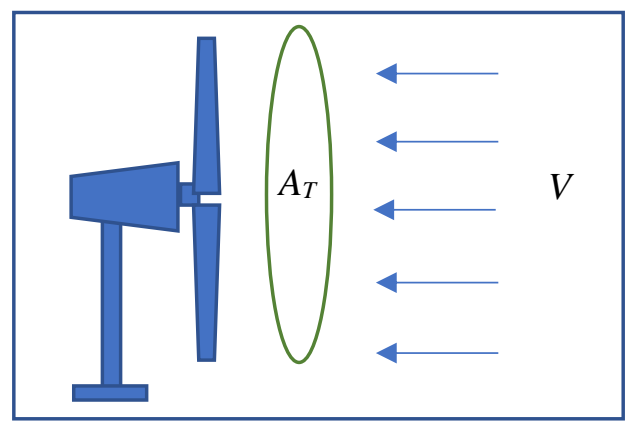

Figura 1 Paquete de aire moviéndose hacia la turbina (Mathew, 2006)

Otros factores como la temperatura, presión atmosférica y altura constituyen la densidad del aire definida por la ecuación:

$\rho_{a}=\frac{m p}{R T}$

siendo $m$ la masa del aire desplazada, $p$ es la presión, $R$ es radio del rotor y $T$ es la temperatura. Una manera práctica para calcular la densidad del aire es:

$\rho_{a}=\frac{353.049}{T} e^{\left(-0.034 \frac{Z}{T}\right)}$

donde las variables a identificar son la temperatura $T$ y la altura $\mathrm{Z}$. El torque esta dado por la expresión:

$$
T=\frac{1}{2} \rho_{a} A_{T} V^{2} R
$$

donde $R$ es el radio del rotor, este se considera como el par teórico máximo en la práctica el rotor solo puede desarrollar una fracción de este límite. La relación entre el torque real desarrollado y el torque teórico se le conoce como coeficiente de torque dado por

$C_{T}=\frac{2 T_{T}}{\rho_{a} A_{T} V^{2} R}$

La potencia desarrollada por un rotor a cierta velocidad del viento depende en gran medida de la velocidad relativa entre la punta del rotor y el viento, por ejemplo:

- Si el rotor está girando a una velocidad muy baja y el viento se acerca al rotor con una velocidad muy alta. En esta condición, a medida que las cuchillas se mueven lenta, una parte de la corriente de aire que se aproxima al rotor puede atravesarla sin interactuar con las cuchillas y, por lo tanto, sin transferencia de energía.
Del mismo modo si el rotor está girando rápido y la velocidad del viento es baja, la corriente del viento puede ser desviada de la turbina y la energía puede perderse debido a la turbulencia y el desprendimiento de vórtices.

En ambos casos, la interacción entre el rotor y el viento el flujo no es eficiente y, por lo tanto, daría como resultado un bajo coeficiente de potencia. A esta relación se le conoce como coeficiente de potencia y es dado por:

$\lambda=\frac{R \Omega}{V}$

Es decir, el coeficiente de potencia depende del radio, velocidades del rotor y viento. En este artículo se propone realizar un estudio del ángulo de ataque del alabe de un aerogenerador para determinar las condiciones óptimas mejorando el coeficiente de potencia y sin poner en riesgo al aerogenerador.

\section{Método}

En la ingeniería es muy recurrente el uso de métodos numéricos para resolver problemas que, por su naturaleza, son difíciles de solucionar algebraicamente. Ejemplo de ello es la integral numérica la cual nos determina el área bajo la curva de un fenómeno físico con buena precisión (Chapra, 2007). En este artículo se utiliza la integral numérica para determinar el gasto energético de una turbina de 3 alabes a 10 grados de inclinación y dos modificaciones (20 y 40 grados).

El método de Simpson es una herramienta muy confiable que consiste en determinar el área bajo la curva de una señal discreta. La herramienta suele ser muy intuitiva y consiste en determinar curvas o polinomios de grado dos, es decir el área bajo la curva descrita por la ecuación:

$A_{s}=A_{1}+A_{2}+A_{3}$

donde $A_{s}$ representa el área total aproximada de la suma de las áreas individuales $A_{1}, A_{2}$ y $A_{3}$, ver figura 2. 


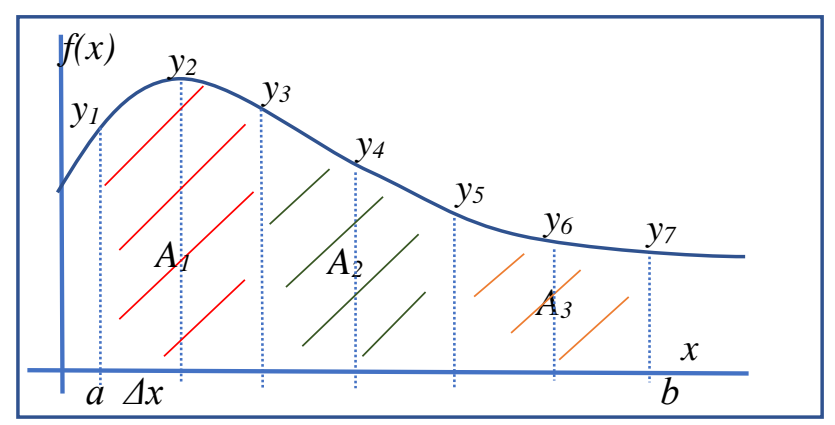

Figura 2 Integración de Simpson con $\Delta x$ constante Fuente: Elaboración Propia

En la figura 2, $f(x)$ corresponde al valor de las velocidades angulares $\left(y_{1}, y_{2}, y_{3}, \ldots, y_{7}\right)$ determinado por el codificador rotatorio en rpm, mientras $\Delta x$ es el paso de iteración constante de la toma de muestra de 5 a $21 \mathrm{~m} / \mathrm{s}$.

Para determinar cada área en cada sección individual, los arcos se pueden aproximar por medio de la forma general de segundo orden de una ecuación de la forma:

$y=a x^{2}+b x+c$

para la primera parábola las constantes desconocidas a, b y c se deben remplazar usando los términos de las velocidades angulares conocidas $y_{1}, y_{2}$ y $y_{3}$ dadas directamente por el codificador rotativo.

Una manera más simple de encontrar el área bajo la curva de la primera parábola consiste en trasladar el origen a $y_{2}$ ver figura 3 .

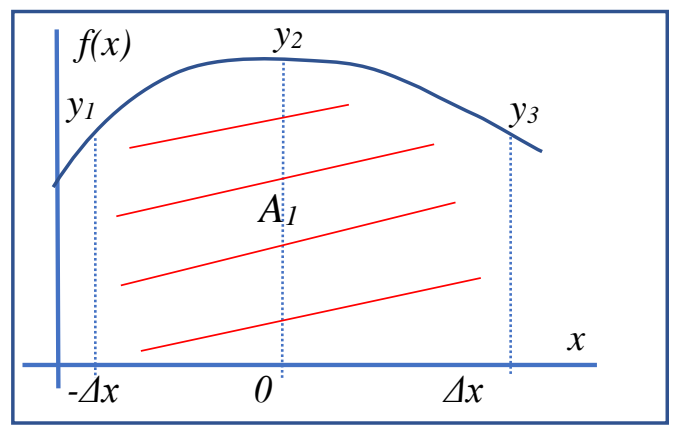

Figura 3 Translación del origen

Fuente: Elaboración Propia

Evaluando en los extremos resulta

$\frac{a \Delta x^{3}}{3}+\frac{b \Delta x^{3}}{3}+c \Delta x-\left(-\frac{a \Delta x^{3}}{3}+\frac{b \Delta x^{3}}{3}-c \Delta x\right)$

donde es fácil mostrar que el gasto energético para el primer intervalo de velocidad del flujo de aire es
$A \rho_{1}=\frac{\Delta x}{3}\left(2 a \Delta x^{2}+6 c\right)$

Evaluando en las velocidades conocidas $\left(-\Delta x, y_{1}\right),\left(0, y_{2}\right)$ y $\left(\Delta x, y_{3}\right)$ en la ecuación (8), se obtiene sistema de ecuaciones:

$\left\{\begin{array}{c}y_{1}+y_{3}=2 a \Delta x^{2}+2 c \\ y^{2}=c\end{array}\right.$

Por lo que el gasto energético para la primera área en términos de las velocidades conocidas del codificador rotatorio queda determinado por:

$A \rho_{1}=\frac{\Delta x}{3}\left(y_{1}+4 y_{2}+y_{3}\right)$

De forma general, la suma del gasto energético de todas las áreas es representado por la ecuación:

$$
\begin{gathered}
\Delta A_{s}=\frac{\Delta x}{3}\left(y_{1}+y_{n-1}+4\left(y_{2}+y_{4}+y_{6}+\cdots+\right.\right. \\
\left.\left.y_{n}\right)+2\left(y_{1}+y_{3}+y_{5}+\cdots+y_{n-1}\right)\right)
\end{gathered}
$$

A través de una herramienta de cómputo es fácil generar el código para determinar el área bajo la curva y, de esta manera, cuantificar el gasto energético de cada aspa.

\section{Resultados}

En la figura 4 se presenta el túnel de viento, al cual se le adaptaron unas aspas movibles para regular el flujo de entrada de aire. Los valores de salida del túnel son de $5 \mathrm{~m} / \mathrm{s}$ hasta $22 \mathrm{~m} / \mathrm{s}$.

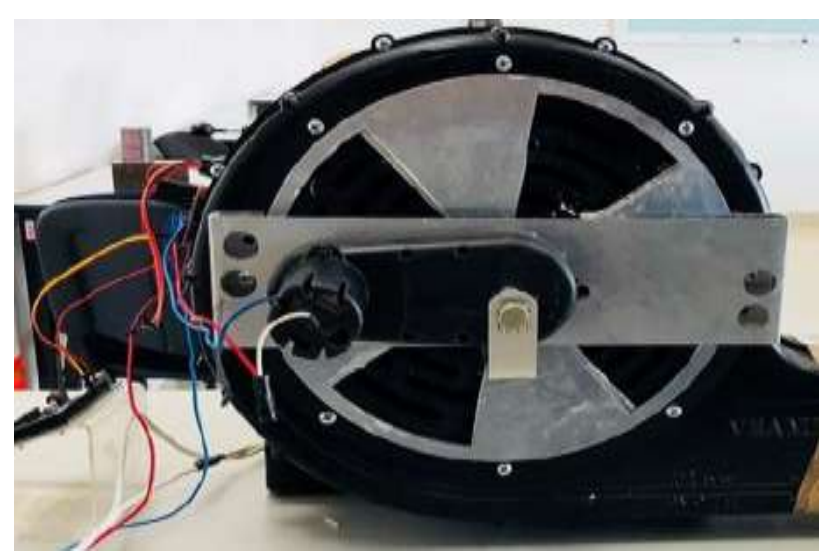

Figura 4 Turbina del túnel de viento

Fuente: Elaboración Propia

En la figura 5 se muestra la cámara de pruebas donde se mide la velocidad de las aspas usando un sensor rotativo (enconder). 


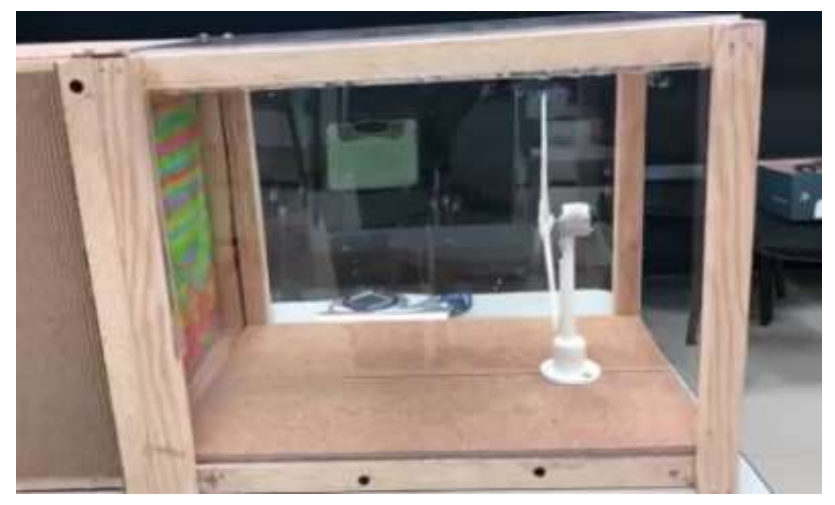

Figura 5 Cámara de pruebas del túnel de viento Fuente: Elaboración Propia

Para realizar el estudio se modeló un aspa de un aerogenerador comercial de baja potencia, el cual se muestra en la figura 6 . Se obtuvo el modelo por medio de un scanner 3D.

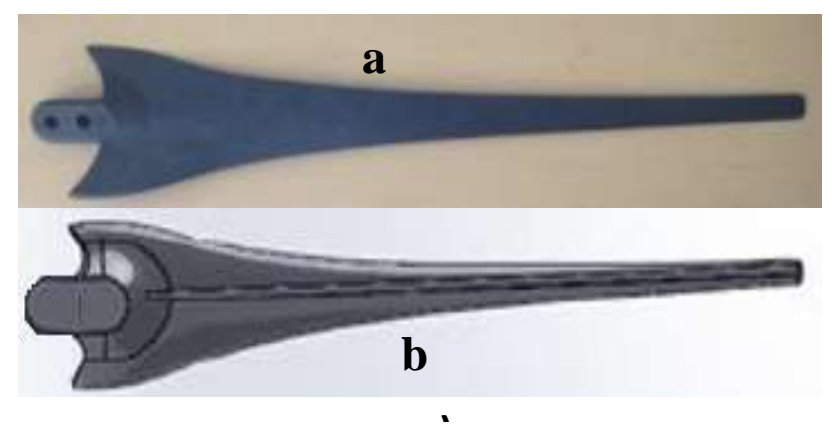

Figura 6 Modelo de aspa

Fuente: Elaboración Propia

Las características principales del aerogenerador de baja potencia son mostradas en la tabla 1.

\begin{tabular}{l|r|}
\hline Potencia a $12 \mathrm{~m} / \mathrm{s}$ & $400 \mathrm{~W}$ \\
\hline Potencia a $8 \mathrm{~m} / \mathrm{s}$ & $200 \mathrm{~W}$ \\
Potencia a $6 \mathrm{~m} / \mathrm{s}$ & $100 \mathrm{~W}$ \\
Potencia a $4 \mathrm{~m} / \mathrm{s}$ & $50 \mathrm{~W}$ \\
Potencia a $3 \mathrm{~m} / \mathrm{s}$ & $0 \mathrm{~W}$ \\
Peso & $6 \mathrm{~kg}$ \\
Velocidad de supervivencia & $42 \mathrm{~m} / \mathrm{s}$ \\
\hline Velocidad del freno mecánico & $14 \mathrm{~m} / \mathrm{s}$ \\
\hline
\end{tabular}

Tabla 1 Características del aerogenerador utilizado Fuente: Elaboración Propia

En la figura 6 se muestra:
a. El original del aerogenerador casero de baja potencia.
b. El modelo obtenido usando un scanner 3D.
c. Mientras en la figura 7 se muestra:
d. El modelo de tres aspas
e. La inclinación original del aspa
f. La inclinación modificada a 20 grados
g. La inclinación modificada a 40 grados

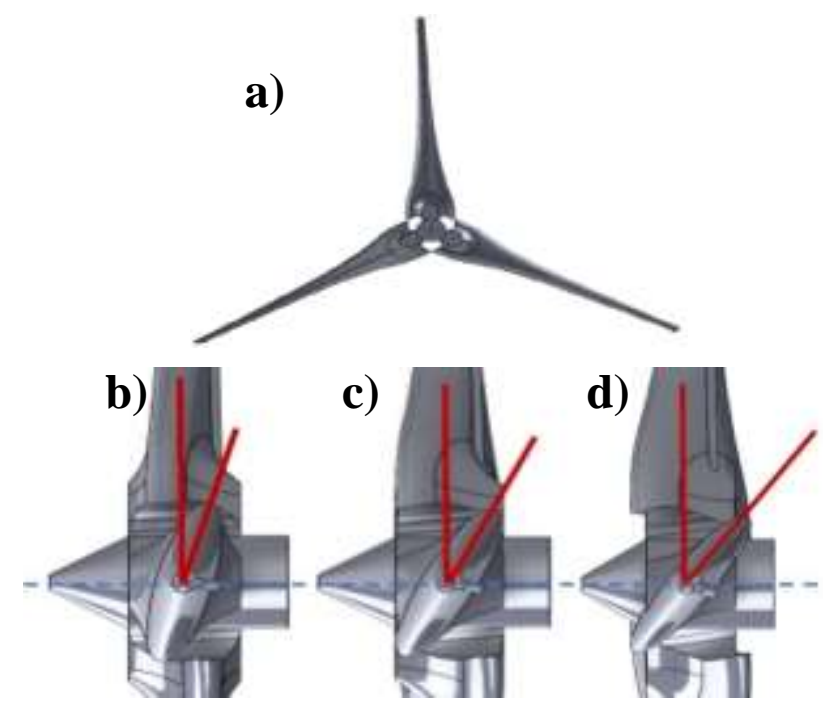

Figura 7 a) sistema de tres hélices, b), c) y d) muestran los modelos del aspa con los ángulos modificados a $10^{\circ}$, $20^{\circ}$ y $40^{\circ}$ respectivamente

Fuente: Elaboración Propia

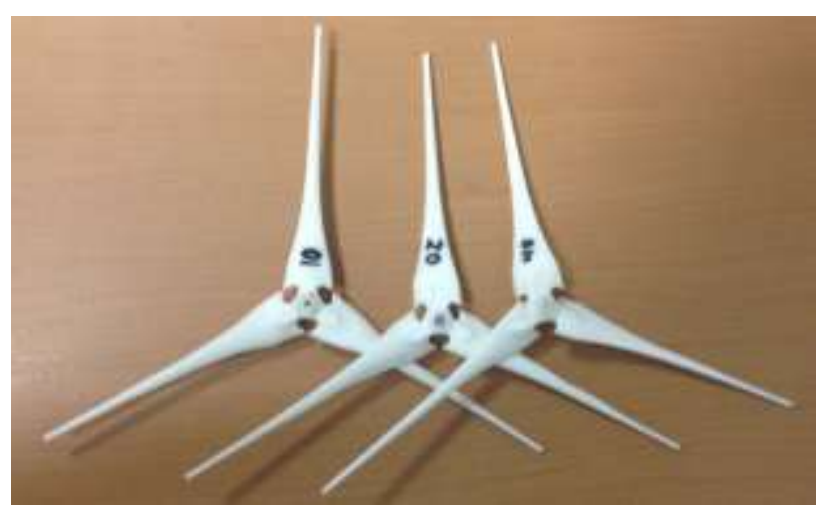

Figura 8 Sistema de tres hélices y modelos modificados del aspa impresos en 3D

Fuente: Elaboración Propia

En la figura 8 se muestra los modelos de las aspas impresas en 3D. En la tabla 3 y figura 9 se muestran los resultados en rpm para las aspas de 10, 20 y 40 grados de inclinación.

\begin{tabular}{|c|r|r|r|}
\hline $\begin{array}{c}\text { Velocidad del } \\
\text { viento } \mathbf{~ m} / \mathbf{s}\end{array}$ & \multicolumn{3}{|c|}{ Inclinación por rpm } \\
\hline 5 & $\mathbf{1 0}^{\circ}$ & $\mathbf{2 0}^{\circ}$ & $\mathbf{4 0}^{\circ}$ \\
\hline 6 & 5 & 9 & 28 \\
\hline 7 & 11 & 19 & 60 \\
\hline 8 & 25 & 48 & 108 \\
\hline 9 & 57 & 88 & 120 \\
\hline 10 & 82 & 122 & 180 \\
\hline 11 & 102 & 145 & 196 \\
\hline 12 & 110 & 178 & 220 \\
\hline 13 & 152 & 210 & 316 \\
\hline 14 & 165 & 220 & 380 \\
\hline 15 & 183 & 225 & 420 \\
\hline 16 & 190 & 266 & 436 \\
\hline 17 & 202 & 275 & 444 \\
\hline 18 & 210 & 310 & 512 \\
\hline 19 & 242 & 323 & 506 \\
\hline 20 & 262 & 365 & 544 \\
\hline 21 & 305 & 388 & 640 \\
\hline
\end{tabular}

Tabla 2 Hélices con diferentes ángulos de inclinación Fuente: Elaboración Propia 


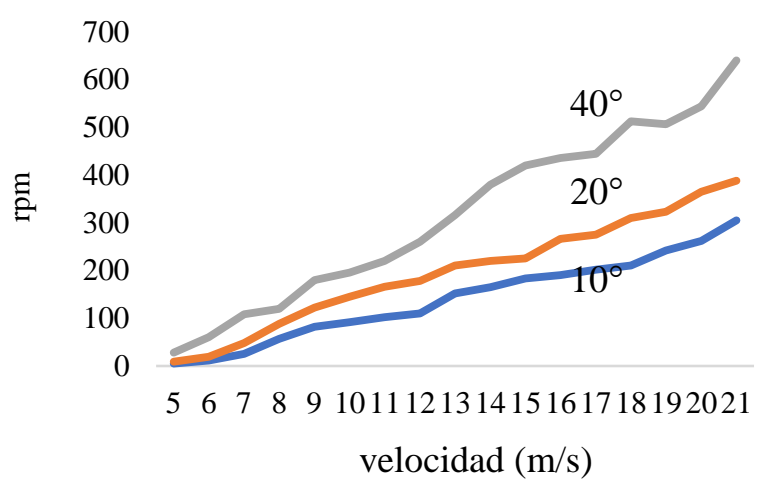

Figura 9 Variación de la velocidad del viento en $\mathrm{m} / \mathrm{s}$ frente a la velocidad angular del buje de la turbina eólica Fuente: Elaboración Propia

Como puede notarse en la tabla 3 y figura 9 , la eficiencia del aspa mejora cuando se realiza la modificación del ángulo de inclinación de 20 y 40 grados. Retomado la ecuación (13), es posible obtener el gasto energético por medio de las integrales numéricas bajo la curva de la figura 6 .

\begin{tabular}{|r|r|}
\hline Ángulo del aspa & Gasto energético \\
\hline $10^{\circ}$ & 2240 \\
\hline $20^{\circ}$ & 3158.5 \\
\hline $40^{\circ}$ & 5036 \\
\hline
\end{tabular}

Tabla 3 Unidades de área de la figura 6 representando el gasto energético

Fuente: Elaboración Propia

La tabla 3 muestra las integrales numéricas de la figura 9 con lo que es fácil obtener el porcentaje de mejora de rendimiento del aspa al realizar la modificación de 20 y 40 grados de inclinación ( $r_{20}$ y $r_{40}$ respectivamente):

$$
\begin{aligned}
& r_{20}=\frac{3158.5\left(r p m * \frac{m}{s}\right)}{2240\left(r p m * \frac{m}{s}\right)} 100 \%=141.0 \% \\
& r_{40}=\frac{5036\left(r p m * \frac{m}{s}\right)}{2240\left(r p m * \frac{m}{s}\right)} 100 \%=224.8 \%
\end{aligned}
$$

\section{Discusión}

Dado que la velocidad del viento no es constante y tampoco se puede asegurar el suministro continuo para producir electricidad, los fabricantes de aerogeneradores de baja potencia diseñan sus aspas para proteger lo mejor posible al equipo contra ráfagas de viento fuertes. Es decir, como los diseñadores no conocen donde se ubicarán sus equipos, plantean el peor escenario de operación haciendo que sus aspas tengan poco rendimiento, soportando así rachas de viento de hasta $40 \mathrm{~m} / \mathrm{s}$.
Pero esto conlleva que sus hélices sean ineficientes a velocidades menores a los $10 \mathrm{~m} / \mathrm{s}$. Como se mostró en el artículo, la modificación del aspa conlleva una mejora en el rendimiento del aerogenerador, pero aumenta considerablemente el par de giro ante rachas de viento superiores a los $14 \mathrm{~m} / \mathrm{s}$, aproximadamente, reduciendo la velocidad de supervivencia significativamente.

Un estudio previo de las condiciones nominales o estándares del clima del lugar donde se ubicarán los aerogeneradores podría, ayudar a elegir mejor el tipo de aspa haciendo más eficiente la generación eléctrica eólica de pequeña potencia y sin poner en riesgo el equipo.

\section{Conclusiones}

La generación de la energía eléctrica por medio de fuentes renovables es una medida que toman los gobiernos y empresas para reducir los efectos de los gases invernadero y reducir costos de operación, consiguiendo ser competitivos en el mercado global.

En este trabajo se presenta un estudio de energía de las aspas de un aerogenerador comercial de pequeño tamaño, en el cual se determinó la velocidad de giro en rpm del aspa a diferentes entradas de velocidad usando un túnel de viento. Posteriormente se realizó la modificación de inclinación del aspa mejorando significativamente su rendimiento.

Como puede verse en el artículo, la velocidad en rpm subió considerablemente con una pequeña modificación del ángulo de inclinación de las aspas, lo cual representa un índice de mejora de rendimiento superior al doble.

Trabajos futuros estarán encaminados optimizar el coeficiente de potencia del aerogenerador, proponiendo un ángulo de ataque de la hélice movible, cambiando el ángulo por medio de un pequeño servomotor. La finalidad es mejorar el rendimiento del aerogenerador a velocidades bajas de entrada del viento y prevenir posibles deterioros en ráfagas altas. Con la información obtenida en este trabajo se podrá justificar la inversión de aerogeneradores de baja potencia para la generación eléctrica. 


\section{Agradecimientos}

A la Universidad Autónoma del Estado de México, UAP Tianguistenco, recursos PFCE 2020.

\section{Referencias}

Akinci, T.C., Seker, S., Guseinoviene, Statistical analysis and Hurst parameter estimation for wind speed in Kirklareli area of Turkey, 8th International Conference and Exhibition on Ecological Vehicles and Renewable Energies, EVER 2013.

Asociación mexicana de Energía Eólica, Noticias AMDEE, México, 2017.

Bakker, A.M.R., van den Hurk, B.J.J.M. Estimation of persistence and trends in geostrophic wind speed for the assessment of wind energy yields in Northwest Europe, Climate Dynamics, 2012.

Baldomero, Mauricio Pablo, Análisis de diferentes álabes de un aerogenerador de eje vertical para oxigenar estanques de peces. Revista Mexicana de Ciencias Agrícolas, 2012.

Chapra, S. C., \& Canale, R. P. Métodos numéricos para ingenieros. McGRAWHILL/INTERAMERICANA EDITORES, S.A. DE C.V, México 2007.

E. Cadenas,a, W. Rivera, Análisis estadístico de mediciones de la velocidad del viento utilizando la técnica de valores desviados, Simposio de Metrología 2008, 2008.

Hernández-Escobedo Q, Espinosa-Arenal F., "Evaluación del potencial eólico para la generación de energía eléctrica en el estado de Veracruz, México", Medellin 2012, Dyna, ISSN 0012-7353.

Mathew Sathyajith. Wind Energy Fundamentals, Resource Analysis and Economics, Springer, New York, 2006, ISBN-13 978-3-540-30905-5 Richmond-Navarro Gustavo, Desempeño de turbinas eólicas Magnus de eje horizontal en función de sus variables geométricas y cinemáticas, Tecnología en Marcha, Vol. 29, N. ${ }^{\circ}$ $1,2016$.

SENER, Prospectiva de Energías Renovables, México, 2016.
Tavner, P. J., \& Spinato, F. Reliability Analysis for Wind Turbines. Wind Energy, 2007. 